\title{
Applications of Nanotechnology in Drug Delivery Systems
}

\author{
Dr.K.Sonamuthu \\ Department of Physics ,J.N.R.M., Port Blair, South Andaman
}

\begin{abstract}
Every so often, a new term comes along that represents an emerging scientific trend. Biotechnology, genetic engineering, tissue engineering, gene therapy, combinatorial chemistry, high throughput screening, and stem cells are some examples of past terms. Recently, nanotechnology has become a popular term representing the main efforts of the current science and technology. Nanotechnology, which is still not a mature technology and thus, more appropriately called nanoscience, usually refers to research at the scale of $100 \mathrm{~nm}$ or less. Nanotechnology is unique in that it represents not just one specific area, but a vast variety of disciplines ranging from basic material science to personal care applications. Thus this technology is very advanced and various researches are a heading in this field very quickly One of the important areas of nanotechnology is "nanomedicine," which, refers to highly specific medical intervention at the molecular scale for diagnosis, prevention and treatment of diseases The nanoparticles are available in various shapes and sizes and these are become very important elements in novel drug delivery systems called nanomedicine. These nanoparticles are developed in appropriate sizes and used such that they target at the targeted places in the body. This development of nanoparticles had brought a revolutionary change in the field of drug delivery systems..

In drug delivery, nanotechnology is just beginning to make an impact. Many of the current "nano" drug delivery systems, however, are remnants of conventional drug delivery systems that happen to be in the nanometer range, such as liposomes, polymeric micelles, nanoparticles, dendrimers, and nanocrystals. Liposomes and polymer micelles were first prepared in 1960's, and nanoparticles and dendrimers in 1970's. Colloidal gold particles in nanometer sizes were first prepared by Michael Faraday more than 150 years ago, but were never referred to or associated with nanoparticles or nanotechnology until recently. About three decades ago, colloidal gold particles were conjugated with antibody for target specific staining, known as immunogold staining. Such an application may be considered as a precursor of recent explosive applications of gold particles in nanotechnology. The importance of nanotechnology in drug delivery is in the concept and ability to manipulate molecules and supramolecular structures for producing devices with programmed functions. Conventional liposomes, polymeric micelles, and nanoparticles are now called "nanovehicles,". Those conventional drug delivery systems would have evolved to the present state regardless of the current nanotechnology revolution.

Cancer is one of the most challenging diseases today, and brain cancer is one of the most difficult malignancies to detect and treat mainly because of the difficulty in getting imaging and therapeutic agents across the blood-brain barrier and into the brain. Many investigators have found that nanoparticles hold promise for ferrying such agents into the brain . Apolipoprotein E was suggested to mediate drug transport across the bloodbrain barrier. Loperamide, which does not cross the blood-brain barrier but exerts antinociceptive effects after direct injection into the brain, was loaded into human serum albumin nanoparticles and linked to apolipoprotein E.
\end{abstract}

Robust angiogenesis underlies aggressive growth of tumors. Therefore, one of the mechanisms to inhibit angiogenesis is to starve tumor cells. Angiogenesis is regulated through a complex set of mediators and recent evidence shows that integrin $\alpha v \beta 3$ and vascular endothelial growth factors (VEGFs) play important regulator roles. The cell adhesion molecules are glycoproteins found on the cell surface that act as receptors for cell-to-cell and cell-to-extracellular matrix adhesion . Recent advancements of the understanding of the cell adhesion molecules has impacted the design and development of drugs (i.e. peptide, proteins) for the potential treatment of cancer, heart and autoimmune diseases. These molecules have important roles in diseases such as cancer, thrombosis and autoimmune diseases such as type-1 diabetes.

There also is an exciting possibility to overcome problems of drug resistance in target cells and to facilitating movement of drugs across barriers such as those in the brain. The challenge, however, remains the precise characterization of molecular targets and to ensure that these molecules are expressed only in the targeted organs to prevent effects on healthy tissues. Secondly, it is important to understand the fate of the drugs once delivered to the nucleus and other sensitive cells organelles. Furthermore, because nanosystems increase efficiency of drug delivery, the doses may need recalibration. Nevertheless, the future remains exciting and wide open.

Keywords: Nanotechnology, nanomedicine, nanoparticles, liposomes, polymeric micelles, nanoparticles, dendrimers, and nanocrystals. Liposomes and polymer micelles,

DOI: $10.7176 /$ APTA/80-02

Publication date:October $31^{\text {st }} 2019$ 


\section{INTRODUCTION}

The definition of nanotechnology is based on the prefix "nano" which is from the Greek word meaning "dwarf". In more technical terms, the word "nano" means 10-9, or one billionth of something. For comparison, a virus is roughly 100 nanometres $(\mathrm{nm})$ in size. The word nanotechnology is generally used when referring to materials with the size of 0.1 to 100 nanometres, Nanotechnology is the study, design, creation, synthesis, manipulation, and application of materials, devices, and systems at the nanometer scale (One meter consists of 1 billion nanometers). It is becoming increasingly important in fields like engineering, agriculture, construction, microelectronics and health care to mention a few. The application of nanotechnology in the field of health care has come under great attention in recent times. There are many treatments today that take a lot of time and are also very expensive. Using nanotechnology, quicker and much cheaper treatments can be developedAs drugs become more complex, and increasingly toxic, new modes of delivery are necessary to transport them to the desired sites in the body. Nanotechnology promises to revolutionize drug delivery by offering targeted administration in addition to more efficient drug dosing. Drugs can be administered orally, topically, intravenously, rectally, or by inhalation. Each method has advantages and disadvantages. When taking a drug by mouth, some of it is decomposed via stomach acids, bile salts, or metabolized by bacteria in the gut. This method is by far the most common and is easiest for the layman to grasp. Topical administration is typically used to fight epithelial infections. Intravenous administration is most commonly used for insulin injections. Rectal administration is helpful with infants or individuals experiencing frequent emesis. Individuals with asthma or respiratory infections may inhale drugs. These options for delivery will likely always be around. However, the drugs administered in these ways will undergo a rapid evolution as nanotechnology inevitably engulfs the pharmaceutical industry. Drugs of today have numerous problems which nanotechnology may solve. When someone takes ibuprofen for a headache, the drug does not target the neurons associated only with the headache, it acts on all neurons in that person's body that it can come in contact with. Nanodrugs will be able to target specific tissues in a body and act only in those tissues. Presently, drug therapy can be very inefficient.

An effective approach for achieving efficient drug delivery would be to rationally develop nanosystems based on the understanding of their interactions with the biological environment, target cell population, target cell-surface receptors [1] changes in cell receptors that occur with progression of disease, mechanism and site of drug action, drug retention, multiple drug administration, molecular mechanisms, and pathobiology of the disease under consideration. It is also important to understand the barriers to drug such as stability of therapeutic agents in the living cell environment. Reduced drug efficacy could be due to instability of drug inside the cell, unavailability due to multiple targeting or chemical properties of delivering molecules, alterations in genetic makeup of cellsurface receptors, over-expression of efflux pumps, changes in signalling pathways with the progression of disease, or drug degradation[2].

\section{Advanced pharmaceutical properties of compounds modified by nanotechnology}

For the determination of drug concentration in the body, if analytical methods are used, the body is sensitive much to detect compounds of less dosage. However nanoparticles introduced to get solution for this crucial problem due to their specific magnetic, chemical, physical, electronic characterizations. The less concentration of drug can be detected by nanoparticles which are having specific magnetic, electric, chemical, physical properties. Thus the pharmacokinetic behaviourand metabolism of these nanoparticles can be measured quantitatively

\subsection{Future biomaterials}

The development of the smart and multifunctional nanomaterials for using in our body will be focussed in the future. Such materials could target specific and desired anatomical structures of the body and the treatment is effective and unique.Nanotechnology in tissue engineering Synthetic polymers used vastly in tissue engineering. $[3-4]$

\subsection{Nanostructured Biomaterials - The Emergence of Silicon}

Silicon is one of the most common elements on Earth, constituting $30 \%$ of the planet's crust, and has been extensively studied by the semiconductor industry. Extremely pure silicon $(>99.999 \%$ pure $)$ is readily available and reasonably inexpensive, and technologies for working with it at the micro and nano levels have been used for decades. Silicon can be made biodegradable by nanostructuring it - an example of a top-down approach — and it safely breaks down in the body into silicic acid, which is found in everyday foodstuffs such as bread and rice. In this form, it can be used as a microparticle carrier for the controlled release of a variety of therapeutics or as a porous membrane in implantable devices, Nanostructured silicon can also be used in a range of other healthcare applications including orthopaedics, tissue engineering and diagnostics. In orthopaedics, for example, porous silicon could potentially be manufactured into orthopaedic implants (pins, screws, etc.) loaded with tissue growth factors, antiinfectives, and anti-inflammatory drugs, and designed to release these drug-loaded silicon can be developed as a coating for traditional medical devices such as metal implants, stents and wound dressings. Perhaps 
the most interesting characteristic of silicon as a biomaterial is its semiconductor properties. The ability to carry an electric charge has obvious potential in treatments where electrostimulation has a positive effect, such as in healing bone fractures and tissue (muscle) repair. But combine this property with the ability to exquisitely control its nanostructure for the controlled release of therapeutics and its proven capabilities in microcircuitry (from the computer chip industry) and there is the potential to develop so-called 'smart' devices. These could potentially be developed as autonomous, self-contained, implantable devices that diagnose disease and deliver the appropriate treatment - the right dose at the right time in the right place. These 'smart' applications of bionanotechnology are a few years away from market yet, but already early smart' device prototypes are in proof of concept stages of development.

\subsection{Drug Delivery through Nanotechnology.}

It is an important question for the society that is whether nanotechnology will really clinical revolution in nanomedicine and drug delivery systems. The answer we can say thatis yes. We have to understand various methods such as solvent.

\section{Design of nanotechnology - based drug delivery Systems}

To achieve efficient drug delivery it is important to understand the interactions of nanomaterials with the biological environment, targeting cell-surface receptors, drug release, multiple drug administration, stability of therapeutic agents and molecular mechanisms of cell signalling involved in pathobiology of the disease under consideration. Several anti-cancer drugs including paclitaxel, doxorubicin, 5-fluorouracil and dexamethasone have been successfully formulated using nanomaterials. Quantom dots, chitosan, Polylactic/glycolic acid (PLGA) and PLGA-based nanoparticles have also been used for in vitro RNAi delivery. Brain cancer is one of the most difficult malignancies to detect and treat mainly because of the difficulty in getting imaging and therapeutic agents past the blood-brain barrier and into the brain. Anti-cancer drugs such as loperamide and doxorubicin bound to nanomaterials have been shown to cross the intact blood-brain barrier and released at therapeutic concentrations in the brain. The use of nanomaterials including peptide-based nanotubes to target the vascular endothelial growth factor (VEGF) receptor and cell adhesion molecules like integrins, cadherins and selectins, is a new approach to control disease progression.

Nanoparticles can be used in targeted drug delivery at the site of disease to improve the uptake of poorly soluble drugs $[5,6]$, the targeting of drugs to a specific site, and drug bioavailability. Several anti-cancer drugs including paclitaxel ,doxorubicin ,5-fluorouracil, and dexamethasone have been successfully formulated using nanomaterials. Polylactic/glycolic acid (PLGA) and polylactic acid (PLA) based nanoparticles have been formulated to encapsulate dexamethasone, a glucocorticoid with an intracellular site of action. Dexamethasone is a chemotherapeutic agent that has anti-proliferative and anti-inflammatory effects. The drug binds to the cytoplasmic receptors and the subsequent drug-receptor complex is transported to the nucleus resulting in the expression of certain genes that control cell proliferation. These drug-loaded nanoparticles formulations that release higher doses of drug for prolonged period of time completely inhibited proliferation of vascular smooth muscle cells.

Presently, drug therapy can be very inefficient. To illustrate, let us imagine a patient with a bacterial infection in her inner or middle ear. The most common method of treatment is a broad spectrum (kills many different kinds of bacteria) antibiotic by mouth for ten days. After the drug enters the stomach, acids degrade some of the drug before it moves to the duodenum (top region of the small intestine). Bile is expelled from the gall bladder into the duodenum, which may further degrade some of the drug before it reaches the small intestine. The small intestine takes the vast majority of the nutrients in our food and puts them into our blood for our bodies to utilize. The same is true for drug absorption; the small intestine is the major site at which drugs are transferred to the blood supply and dispersed throughout the body. The problem with this method is that the antibiotic not only kills the bacteria in the ear, it may also kill bacteria in the sinuses, throat, stomach, intestines, colon, and vaginal epithelium. This poses a risk of pathogenic bacteria colonizing those tissues when the drug treatment subsides. Antibiotics are not the only drugs that pose problems associated with undesired absorption patterns. Selectivity of drug absorption is so poor that massive dosages are needed often creating worse problems than the ones the drug is intended to alleviate or cure.

\subsection{Nanotechnology and Drug bioavailability}

Drug bioavailability is a related problem with potential nanotechnology solutions. Again, biodegradable polymer capsules show promise. Hydrophobic drugs such as paclitaxel or 5-fluorouracil can be encapsulated in polymers or liposomes with nanoscale cavities that improve drug absorption and bioavailability. The opportunity exists to systematically look at both successful and failed drugs to see which ones might benefit from novel delivery vehicles. In some cases, reformulation of a drug with smaller particle size may improve oral bioavailability. Nanotechnology is being used to create new diagnostic pharmaceuticals for use in medical imaging. The class of 
compounds known as superparamagnetic iron oxides (SPIOs), also known as monocrystalline iron oxide nanoparticles, or MIONs, have shown promise for a number of magnetic resonance (MR) imaging applications both as naked particles and as magnetic labels

\subsection{Nanoparticle-mediated delivery of siRNA}

Short interfering RNA (siRNA) is emerging as a robust method of controlling gene expression with a large number of applications. Translation of nucleic acid-based therapy to clinical studies will require significant advances in the delivery system. Quantum dots (QD) have been used to monitor RNAi delivery. PLGA and PLA based nanoparticles have also been used for in vitro RNAi delivery. Although there has been some success in the delivery of siRNA using various nanomaterials, tracking their delivery and monitoring their transfection efficiency is difficult without a suitable tracking agent or marker. Designing an efficient and self-tracking transfection agent for RNA interference is a big challenge.

\section{Disease Diagnosis and Prevention}

4.1 Diagnosis and Imaging

Nanobiotech scientists have successfully produced microchips that are coated with human molecules. The chip is designed to emit an electrical impulse signal when the molecules detect signs of a disease. Special sensor nanobots can be inserted into the blood under the skin where they check blood contents and warn of any possible diseases. They can also be used to monitor the sugar level in the blood. Advantages of using such nanobots are that they are very cheap to produce and easily

\section{2 frying tumors}

Nanomaterials have also beeninvestigated into treating cancer. The therapy is based on "cooking tumors" principle. Iron nanoparticles are taken as oral pills and they attach to the tumor. Then a magnetic field is applied and this causes the nanoparticles to heat up and literally cook the tumors from inside out Cancer Cooker- Triton BioSystems is developing an anticancer therapy using antibodycoated iron nanoparticles

\subsection{Preventing diseases}

Heart-attack prevention Nanobots can also be used to prevent heart-attacks. Heart-attacks are caused by fat deposits blocking the blood vessels. Nanobots can be made for removing these fat deposits The following figure shows nanobots removing the yellow fat deposits on the inner side of blood vessels.

\section{Nanorobots}

The somewhat speculative claims about the possibility of using nanorobots in medicine, advocates say, would totally change the world of medicine once it is realized. Nanomedicine would make use of these nanorobots (e.g., Computational Genes), introduced into the body, to repair or detect damages and infections. According to Robert Freitas of the Institute for Molecular Manufacturing, a typical blood borne medical nanorobot would be between 0.5-3 micrometres in size, because that is the maximum size possible due to capillary passage requirement. Carbon would be the primary element used to build these nanorobots due to the inherent strength and other characteristics of some forms of carbon (diamond/fullerene composites), and nanorobots would be fabricated in desktop nanofactories specialized for this purpose. Nanodevices could be observed at work inside the body using MRI, especially if their components were manufactured using mostly $13 \mathrm{C}$ atoms rather than the natural $12 \mathrm{C}$ isotope of carbon, since $13 \mathrm{C}$ has a nonzero nuclear magnetic moment. Medical nanodevices would first be injected into a human body, and would then go to work in a specific organ or tissue mass. The doctor will monitor the progress, and make certain that the nanodevices have gotten to the correct target treatment region. The doctor will also be able to scan a section of the body, and actually see the nanodevices congregated neatly around their target (a tumor mass, etc.) so that he or she can be sure that the procedure was successful.

\section{Nanonephrology}

Nanonephrology is a branch of nanomedicine and nanotechnology that deals with 1) the study of kidney protein structures at the atomic level; 2) nanoimaging approaches to study cellular processes in kidney cells; and 3) nano medical treatments that utilize nanoparticles and to treat various kidney diseases. The creation and use of materials and devices at the molecular and atomic levels that can be used for the diagnosis and therapy of renal diseases is also a part of Nanonephrology that will play a role in the management of patients with kidney disease in the future

\subsection{Targeting cancer cells with nanoparticles}

Cancer is one of the most challenging diseases today, and brain cancer is one of the most difficult malignancies to detect and treat mainly because of the difficulty in getting imaging and therapeutic agents across the blood-brain 
barrier and into the brain. Many investigators have found that nanoparticles hold promise for ferrying such agents into the brain. Apolipoprotein E was suggested to mediate drug transport across the blood-brain barrier. Loperamide, which does not cross the blood-brain barrier but exerts antinociceptive effects after direct injection into the brain, was loaded into human serum albumin nanoparticles and linked to apolipoprotein E. Mice treated intravenously with this complex induced antinociceptive effects in the tail-flick test. The efficacy of this drug delivery system of course depends upon the recognition of lipoprotein receptors. Kopelman and colleagues designed Probes Encapsulated by Biologically Localized Embedding (PEBBLE) to carry a variety of unique agents on their surface and to perform multiple functions .

\section{Medical Treatments by Nanotechnology relating to cancer.}

Nanoshells may be used to concentrate the heat from infrared light to kill cancer cells with minimal damage to surrounding healthy body cells. A nanoshell consists of a gold coated metal shell and a non-conducting silica centre and the shell is able to absorb nearby infrared laser energy emitted by a source. The nanoparticles are injected into the bloodstream where they travel to the cancerous growth. Nanospectra Biosciences has developed such a treatment using nanoshells illuminated by an infrared laser that has been approved for a pilot trial with human patients. The advantage of using nanoshells to treat cancerous tumours is that they can destroy cancerous cells but do not excessively damage normal cells in the process. There is an advantage over chemotherapy, which destroys both cancerous and normal cells. The drugs used in chemotherapy are nt able to target the cancerous growth specifically, and the damage of healthy body cells can result in secondary conditions such as immune system suppression and hair loss, whereas nanoparticles have the potential to be able to target only the cancer cells, so prove to be a more harmless treatment. Another advantage is that this procedure is much less invasive than current common treatments such as surgically resecting the tumour as shown in Fig-1.

\subsection{Medical Diagnosis:}

Quantum Dots may be used in the future for locating cancer tumours in patients. Current testing has been done on mice, where the quantum dots have been observed to give off a detectable fluorescence when stimulated by UV light, hence indicating the position of the tumour. By combining different sized quantum dots within individual granules, granules that release distinctive colours and intensities of light, after stimulation by UV light, can be created. The large variety of quantum dots can give scientists the potential to make them into many different granules so high numbers of DNA sections can be coded for and recognized. Cancerous tumours contain many different types of mutating DNA, so therefore quantum dots can be used to identify different kinds of cancer, reducing the requirement for an invasive biopsy which can be uncomfortable for the patient, especially when brain tumours are concerned and a craniotomy has to be performed with the patient under a general anaesthetic. Scientists have experimented by injecting PEG coated quantum dots in to the bloodstream of a mouse and the effect of the surface coating on the length of time of circulation in the blood was observed. It was found that the circulation time was much longer in comparison to organic dyes which had a short half life. Organic dyes also can only come in certain amount of colours and are susceptible to photo bleaching so therefore cannot always be reliable. However, quantum dots are highly resilient against photo bleaching and come in a diverse range of colour shades(once activated by UV light), allowing a great range of cells to be identified . The quantum dots also have high contrast which makes them able to recognize the smallest of tumours, allowing early detection whereas currently used radioactive markers have low specificity meaning that they cannot always detect small areas of affected tissue. The long circulation feature can be explained by the unique structure of the quantum dots, as they are large enough to avoid renal filtration by the kidneys, but small and hydrophilic enough to slow down non specific binding of proteins.

\subsection{Nanotechnology involving diagnostic methods for brain cancers.}

Currently, gadolinium chelate contrast-enhanced MRI imaging is the main method of detection and diagnosis of pre-operative brain tumours due to the fact that it is not invasive and has high resolution and contrast. However, the imaging quality may still be seen as limited in its ability to accurately indicate the boundaries and distinctions between the tumour cells and healthy brain cells, and give a very accurate idea of the volume taken up by the tumour, due to surrounding edema and diffusion of contrast agents from the tumour cells. Therefore, it may be said that a patient's recovery is sometimes hindered by the inability to accurately locate tumour cells and distinguish them from healthy tissue. During surgery, it may be difficult to resect the entire tumour, if it is tough to distinguish between cancer cells and normal cells.

Scientists have done research into using superparamagnetic iron oxide nanoparticles as MRI contrast agents in order to increase the visual distinction between cancer and normal brain tissue. The nanoprobe is made of up an iron oxide core, coated with PEG (polyethylene glycol), and joined together with the targeting agent chlorotoxin . Chlorotoxin is a scorpion derived 36 amino acid peptide, which has high selectivity for gliomas and other brain cancers, once it has been injected into the bloodstream. It has also been show to be able to bind to medullablastoma 
(another brain cancer type), prostatecancer and intestinal cancer. Non targeting nanoparticles are those which do not contain agents which are able to specifically target and bind to the cancer cells, but chlorotoxin is able to bind to cancer cells and therefore offer better contrast MRI imaging and enhance the quality, due to the fact that the chlorotoxin is able to recognise the molecular markers present in cancer cells. The magnetic property of iron oxide enhances the images from the MRI scan, allowing areas of different densities to be more clearly differentiated between and giving an advantageous increased sensibility in the ability of MRI scans to detect small tumours. This gives the opportunity for a better identification of brain tumours and also gives neurosurgeons more information regarding the tumour so that they are able to perform a more thorough resection of the cancer. It reduces the chances of damage to surrounding tissue [6].

\subsection{Nanotechnology Drug Targeting Glioma Brain Tumours}

The molecular structure of the most aggressive type of brain tumours, the glioblastoma multiforme (gliomas). Gliomas are malignant cancerous tumours and are very difficult to treat. They tend to spread rapidly by metastasis to healthy brain tissue and have been found to reappear in locations which make their surgical removal a very difficult task. In $80 \%$ of patients with gliomas, they cause seizures as they release toxic chemicals to kill healthy nerve cells, and can cause paralysis. They are resistant to chemotherapy and radiotherapy and the brain itself is protected by a blood-brain barrier and the immune system has mechanisms which thwarts most therapies. To make matters worse, they are the most common form of brain cancer with a high mortality rate and median survival rate of about 14 months.

The scientists discovered that there is a type of protein called laminin-411 that plays a major part in the tumour's tendency to create new blood vessels to aid its growth and development and metastasis. The ability of technology to block this protein could lead to an advance in glioma treatment. Using nanotechnology, a nanobioconjugate drug was created by the scientists that could be given to a patient intravenously and could then be carried in the blood to the glioma. It is comprised of nanoparticles of 20 to $30 \mathrm{~nm}$ in size, is based on a highly purified form of polymalic acid derived from the single cell organism Physarum polycephalum. It was created with the specific property of being able to permeate the tumour cells, and enter the endosomes. As endosomes mature, they become acidic and the chemical constituent of the drug is able to act at this point and break the endosomes' membranes. The tumour cells' production of laminin-411 is able to be blocked, preventing the protein from being formed and production of new tumour vessels. A bonus is that the drug is not toxic to healthy brain cells, so therefore do not share the disadvantages of chemotherapy. Studies in mice showed that large amounts of the drug could accumulate in the tumours and drastically reduce the growth of the tumour. Tumours affected by the drug became $90 \%$ smaller than without the drug. The drug is able to enter the brain and target the cancer specifically. Due to its strong chemical bonds, the components of the drug are able to avoid damage or separation in tissues or blood plasma during their transport through the human system. The anti tumour component is able to activate directly inside tumour cells, which is different from other nanomedicine drugs that activate at the site of tumour, not in the tumour cells themselves[7,8]. The nanobioconjugate drug has been specifically designed to be able to produce a chain of biochemical events such as releasing the anti tumour drugs at the right place and time, homing in on the tumour cells directly, permeating the walls of blood vessels and dismantling processes which help the blood supplying vessels to the tumour grow. When the nanobioconjugate has accomplished its tasks, the body digests it completely, leaving no harmful residue. It is changed into carbon dioxide and water which is not toxic to healthy cells. Another advantage is that doesn't stimulate the immune system to produce reactions such as coughs or rashes or any other systems. Therefore, it is predicated that human trials of the drug may begin soon.

\subsection{Promises and challenges of nanomedicine}

Nanotechnology has its own importance in medical field. It has some challenges such as use of nanotechnology in vaccines like Hepatitis B vaccine. Currently researches are going on to overcome the problems associated with nanotechnology [9].

\section{Applications and hazards of nanomedicinal drug delivery systems}

Highly specific drug targeting, decrease in toxicity during therapeutic effect, biocompatibility and safety is high, fast manufacturing of desired drugs.HazardsIn vivo: pulmonary inflammation in the rat, cause more lung tumors than fine particles in rat chronic studies, affects immune responses.Invitro: Affects rolling in hepatic tissue, inhibits macrophage phagocytosis, mobility, killing, platelet aggregation[10].

\subsection{Future impact of nanomedicine in human life and dentistry}

Nanotechnology is a part of a predicted future in which dentistry may become more effective. Research is going on to find the way to make use of nano or micro scopic moieties to perform tasks that are done by equipment. Treatment options will become numerous with nanomedicine and exciting $[11,12]$ 


\section{CONCLUSION}

It appears that nano drug delivery systems hold great potential to overcome some of the barriers to efficient targeting of cells and molecules in inflammation and cancer. There also is an exciting possibility to overcome problems of drug resistance in target cells and to facilitating movement of drugs across barriers such as those in the brain. The challenge, however, remains the precise characterization of molecular targets and to ensure that these molecules are expressed only in the targeted organs to prevent effects on healthy tissues. Secondly, it is important to understand the fate of the drugs once delivered to the nucleus and other sensitive cells organelles. The ability of nanoparticles to be able to specifically target cancerous brain tumours gives a promising possibility of minimising damage to healthy body cells, which is a disadvantage of some current treatment methods such as chemotherapy resulting in a great physical and emotional impact on the patient receiving treatment. Furthermore, because nanosystems increase efficiency of drug delivery, the doses may need recalibration. Nevertheless, the future remains exciting and wide open.

\section{REFERENCE}

1. Won Hyuk Suh, Kenneth S. Suslick, Galen D. Stucky and Yoo-Hun Suh.

Nanotechnology nanotoxicology andneuroscience. Prog Neurobiol.2009;87(3):133-170.

2. Xing-Jie Liang, Chunying chen, Yuliang Zhao, Lee Jia and Paul C. Wang.Biopharmaceuticals and therapeutical potentials of engineered nanomaterial.

3. Hagit Bar, Iftach Yacoby and Itai Benhar.Killing cancer cells by targeted drug carrying phage nanomedicines. BMC Biotechnology. 2008;8:37.

4. Nathaniel Huebsch, David J. Mooney. Nature. 2009;462(7272):426-432.

5. Jason H. Sakamoto et al. Enabling individualized therapy through nanotechnology. Pharmacol Res.2010;62(2):57-89.

6. Shihua Jin MD and Vinod Labhasetwar PHD. Nanotechnology in urology. Urol Clin North Am. 2009;36(2):179-188.

7. Suegei V Vinogradov. Polymeric nanogel formulations of nucleoside analogues.Expert Opin Drug Deliv. 2007;4(1):5-17.

8. Michelle Pautler and Sara Brenner.Nanomedicne; promises and challenges for the future of public health.International Journal of Nano medicine.2010;5:803-809.

9. Wim H De Jong and Paul JA Borm. Drug delivery and nanoparticles, applications and hazards. International Journal of Nanomedicine. 2008:3(2):133-149.

10. Mallanagouda Patil, Dhoom Sing Mehta and Sowjanya Guwa. Future impact of nanotechnology on medicine and dentistry. J Indian Soc Periodontol. 2008;12(2):34-40.

11. Mette Ebbesen and Thomas G Jensen.Nanomedicine: Techniques, potentials and

ethical implications. Hindawi Publishing Corporation Journal of Biomedicine and

Biotechnology. 2006:1-11.

12. Kyle Phua and Kam W Leong. Microscale oral delivery devices incorporating nanoparticles. Nanomedicine (Lond).2010;5(2):161-163.

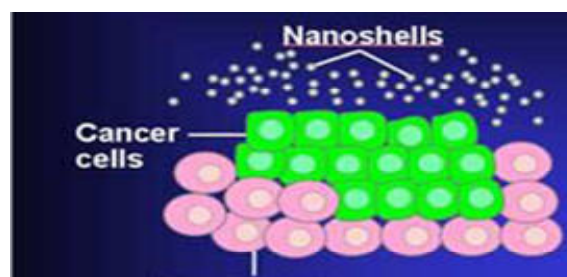

Healthy cells

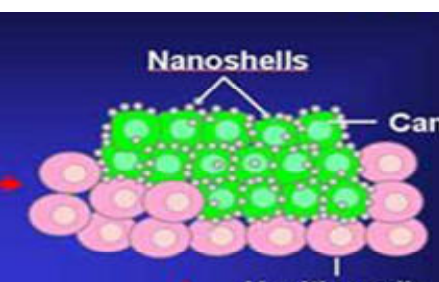

Healthy cells

Fig-1

Near-infrared light
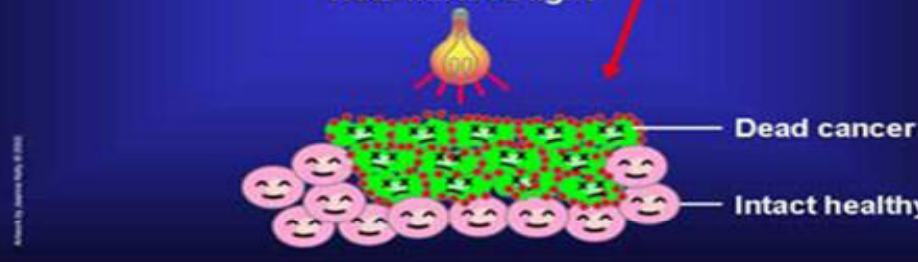

Nano shells as cancer therapy 